\title{
Amenable mortality as an indicator of healthcare quality - a literature review
}

Sophia Kamarudeen Office for National Statistics

\section{Abstract}

\section{Background}

In 2008, the Office for National Statistics (ONS) suggested that research should be undertaken into whether amenable mortality - deaths considered avoidable due to medical intervention - could be used as an indicator of healthcare quality. The aim of this paper is to review the literature on amenable mortality in order to determine the extent to which the observed fall in amenable mortality is due to the healthcare system.

\section{Methods}

The literature reviewed covers mainly the Organisation for Economic Co-operation and Development (OECD) countries and can be broadly categorised into: (a) trends across OECD countries (b) pooled time series cross-country analyses of OECD countries and (c) cross-section or time series analyses within countries.

\section{Results}

There is no consensus in the literature on exactly what constitutes amenable mortality, thereby making the concept in itself imprecise. However, there has been a fall in amenable mortality in the past few decades in most OECD countries including the UK. Since the fall in amenable mortality has been at a faster rate than that of 'unavoidable' mortality, some studies have directly attributed this to the healthcare system. Others have tested the relationship between healthcare outcomes and healthcare inputs to determine the magnitude and direction of the relationship.

No study has explicitly used a healthcare activity or quality variable in their analyses. This implies that the evidence that amenable mortality is an indicator of healthcare quality is far from overwhelming or clear. 


\section{Conclusions}

At this stage, it is premature to use amenable mortality in ONS's healthcare output calculations. We welcome comments from those interested in this field, and suggestions to improve understanding in this area.

\section{Editor's note}

This article originally appeared on the ONS website on 30 March 2010 at http://www.statistics.gov.uk/cci/article.asp?id=2397. It is reproduced here to disseminate the findings more widely and make the content readily available to readers of HSQ. As the original article was quality assured by selected members of the UK Centre for the Measurement of Government Activity (UKCeMGA) quality assurance panel for Healthcare, additional peer review was not considered necessary before publication in HSQ. 


\section{Contents}

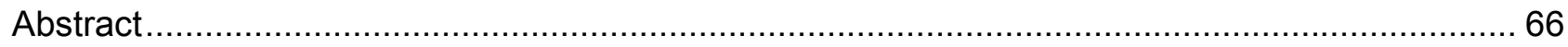

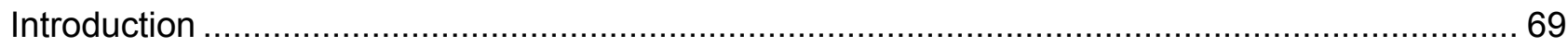

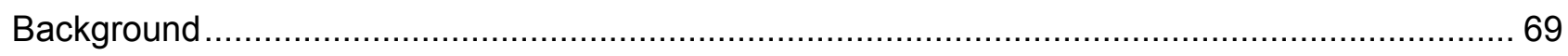

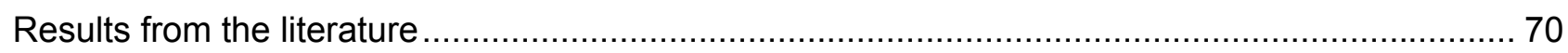

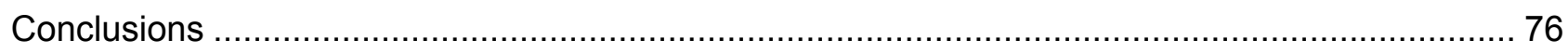

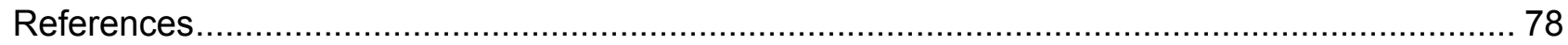

\section{List of Figures}

Figure 1 Decline in amenable mortality amongst males and females aged $0-74$. Nineteen OECD countries, 1997/98-2002/03 .............................................................. 71

Figure 2 Decline in amenable, preventable and unavoidable mortality amongst males and females aged 0-74 in England and Wales, 1993-2005 …................................ 72 


\section{Introduction}

The ONS is responsible for producing estimates of public service output and productivity, and therefore publishes measures of healthcare output and productivity for the UK (ONS 2010; ONS 2008; ONS 2006; ONS 2004). Healthcare output is measured using healthcare activity, and adjusted for quality using the following indicators:

- short-term survival and health gain following treatment in hospital, including health effects of shorter waiting time

- outcomes from primary medical care

- assessment of patient experience

As part of the work to improve the measure of healthcare output, ONS proposed an investigation of amenable mortality as an indicator of healthcare quality (ONS 2008).

\section{Background}

In recent decades in the UK, the rate of amenable mortality, that is deaths considered avoidable due to medical intervention, have fallen. In England and Wales, the age-standardised mortality rate (takes into account differences in the age structure of populations and allow comparison over time and between sexes) for all causes considered amenable to medical intervention decreased by around 43 per cent for males and 38 per cent for females between 1993 and 2005 (Wheller et al. 2007).

This raises an obvious question - how much, if any, of the observed decline in amenable mortality can be attributed to the healthcare system? The answer, however, is not obvious since apart from healthcare, there is a wide range of socio-economic and lifestyle factors that influence health outcomes.

The Department of Health $(\mathrm{DH})$ in a recent paper on quality adjustments to healthcare output (DH 2007) estimated what would happen if the entire observed decline in amenable mortality in England were due to the National Health Service (NHS). DH valued each life year saved at $£ 30,000$ to calculate the addition to NHS output. They found that, between 2000 and 2005, the resulting increase in NHS output would equal approximately $£ 2.9$ billion and add approximately 1 per cent per annum to the output series. DH notes however that:

- there is no evidence that the decline in amenable mortality can be attributed entirely to the NHS

- amenable mortality might be declining (i) due to an increase in NHS activity (ii) an improvement in NHS quality. If the entire fall in amenable mortality is attributed to the NHS and thereby added to the output series, this could result in double counting since NHS activity is already included in the output series

- current quality indicators, especially survival after hospital treatment, might already be picking up the impact of declining amenable mortality on healthcare output

In order to include amenable mortality in ONS's healthcare output calculations, there is a need to validate the relationship between amenable mortality and healthcare. In other words, what proportion of the decline in amenable mortality can be attributed to the healthcare system and, 
more importantly, is it possible to assess how much of the decline is due to an increase in activity and how much to an improvement in quality?

\section{Aim of the paper}

The primary aim of this paper is to review the literature on amenable mortality in order to determine the extent to which the observed fall in amenable mortality is due to the healthcare system. This is in line with the principle set out in the Atkinson Review (2005) which states that 'the output of the government sector should in principle be measured in a way that is adjusted for quality, taking account of the attributable incremental contribution of the service to the outcome.'

This paper also identifies gaps in the literature and suggests directions for future research. The implications of the findings for the work of the ONS on healthcare output and productivity are also discussed. Only studies that focus on OECD countries are covered.

\section{Results from the literature}

\section{What constitutes amenable mortality is not clear}

Amenable mortality can broadly be defined as deaths occurring before age 75 from causes that are considered amenable to medical intervention (Wheller et al. 2007). Examples include breast cancer, cancer of colon and rectum, leukaemia, gastric and duodenal ulcer, and hypertensive diseases. There is, however, no consensus in the literature on exactly what constitutes amenable mortality (Wheller et al. 2007; Nolte and McKee 2004). Advances in medical care mean that diseases that are considered amenable to medical intervention keep changing over time. Additionally, the 'cut off' age can vary depending on the condition in question. For instance, some authors set the age limit for diabetes mellitus under 50 because the preventability of deaths at older ages from diabetes remains controversial, while the age limit for whooping cough and measles might be set under 15 since deaths other than in childhood from these diseases are likely to reflect the presence of other disease processes (Nolte and McKee 2008).

While some studies focus on deaths amenable to medical intervention, others also include deaths considered preventable due to primary care or public health policies (often termed 'preventable mortality') such as lung cancer, illicit drug use disorders, transport accidents, and Hepatitis B. With preventable mortality however, individual lifestyle factors and socio-economic factors might play a proportionately larger role when compared to healthcare factors, thereby making it harder to separate the impact of healthcare from other factors. Nevertheless, the role of public health policies in mitigating deaths from preventable causes cannot be ignored altogether.

The literature also uses the term 'avoidable mortality' which may refer to (i) amenable mortality or (ii) both amenable and preventable mortality. This paper is not restricted to studies that cover amenable mortality but also includes those that discuss preventable mortality. 


\section{Trends in amenable mortality}

The vast majority of the literature on amenable mortality discusses trends across or within countries. As mentioned before, this review is limited to OECD countries with a particular focus on the UK.

\section{Declining rates of amenable mortality across OECD countries}

Nolte and McKee (2008) carried out a comprehensive study of amenable mortality in 19 OECD countries between 1997/98 and 2002/03. They calculated the age-standardised death rates among males and females from selected causes and found a clear decline in amenable mortality in all countries (Figure 1). Among males, the average fall was 17 per cent, while for females it was 14 per cent. The USA showed the smallest decline, 4 per cent among males and just over 5 per cent among females. In the UK, the decline was 22 per cent for males and 20 per cent for females. It is important to acknowledge that the starting levels of mortality in these countries are different. However, the main point here is the observed decline in amenable mortality rates.

\section{Figure 1 Decline in amenable mortality amongst males and females} aged 0-74. Nineteen OECD countries, 1997/98-2002/03

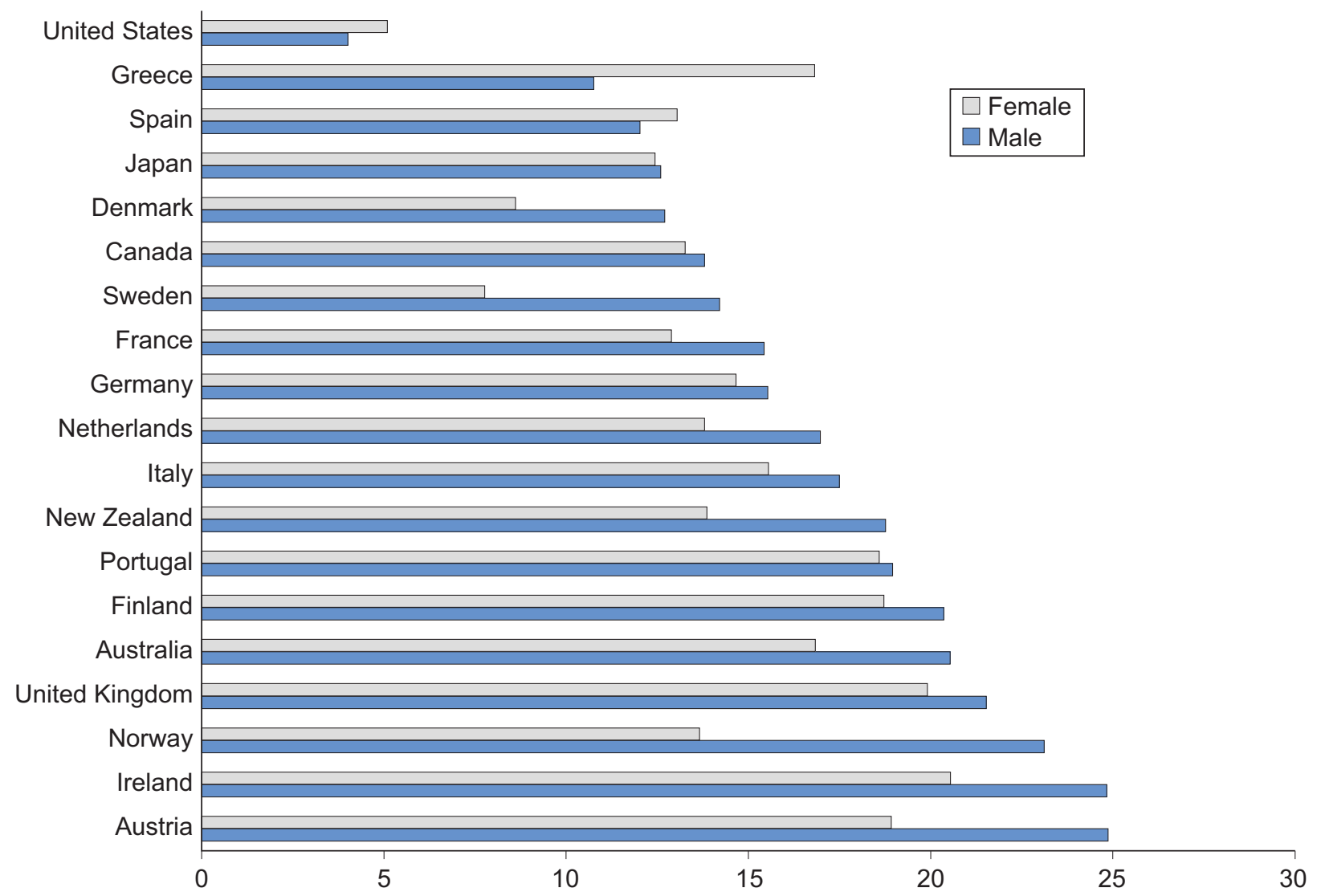

Source: Nolte and McKee (2008) 


\section{Declining rates of amenable mortality in England and Wales}

Wheller et al. (2007) measured the rate of avoidable mortality in England and Wales between 1993 and 2005 and concluded that there was a fall in both amenable and preventable mortality.

Figure 2 shows the decline in amenable, preventable and unavoidable mortality using the Page, Tobias and Glover (2006) definition of avoidable mortality. Between 1993 and 2005, mortality from amenable causes decreased by 46 per cent and 41 per cent for males and females respectively; from preventable causes by 18 per cent for males and 8 per cent for females; and unavoidable causes by 9 per cent among males and 5 per cent among females.

The authors conclude that the 'decreases in mortality from avoidable causes are unlikely to simply be the result of a general decrease in mortality rates and that medical interventions are likely to have contributed positively to reductions in avoidable mortality, as are public health initiatives, though perhaps to a lesser extent'.

\section{Figure 2 Decline in amenable, preventable and unavoidable mortality amongst males and females aged 0-74 in England and Wales, 1993-2005}

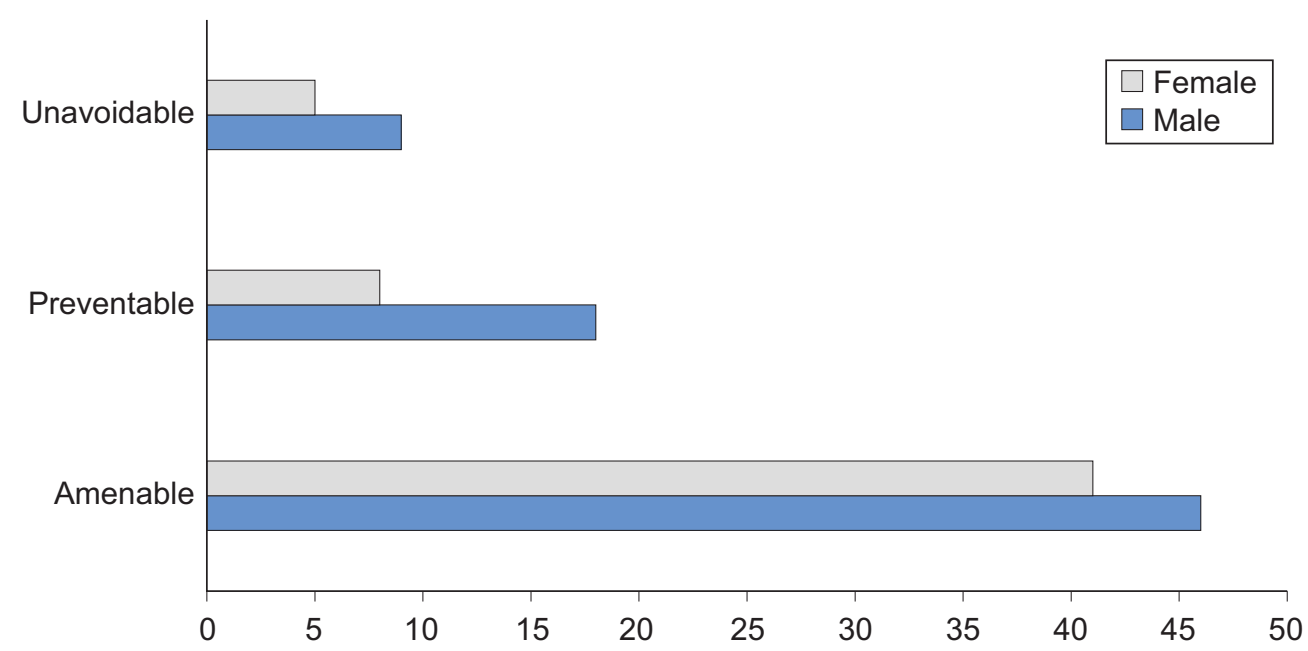

\section{What the trends tell us}

There is a clear decline in amenable mortality in most OECD countries, including the UK. Some studies have directly attributed the decline to the healthcare system. However, Nolte and McKee (2008) point out that 'amenable mortality should not be mistaken as a definitive evidence of differences in effective healthcare but rather as an indicator of the potential weaknesses in healthcare that can be investigated in more depth.' 
The following sections will discuss those studies that have tested the magnitude and direction of the relationship between healthcare inputs and healthcare outcomes. The results will determine how much, if any, of the fall in amenable mortality can be attributed to the healthcare system.

\section{Empirical evidence of the relationship between health outcomes and inputs}

The most common method employed to test the relationship between health outcomes (for example, life expectancy) and health inputs is a health production function. Studies reviewed in this section can be split into (i) pooled time series cross-country analyses of OECD countries and (ii) cross-section or time series analyses within countries. Not all studies reviewed have tested the relationship between healthcare inputs and amenable mortality per se - some have focused on the impact of healthcare on mortality in general.

The equation in Box 1 shows a typical health production function estimation. In order to determine if amenable mortality can be attributed to the healthcare system, the set of medical variables in the equation should ideally include indicators or measures of healthcare activity and healthcare quality.

\section{Box $1 \quad$ Health Production Function}

$$
H_{i t}=\alpha_{i}+\beta M_{i t}+\gamma E_{i t}+\varepsilon i t
$$

$H$ is a measure of health outcome;

$M$ captures medical or healthcare variables;

$E$ is a list of non-medical variables;

subscripts $i$ and $t$ represent country and time;

the sign on $\beta$ and $\gamma$ gives the direction of the relationship between the respective health input and health outcome, and their values give the magnitude;

$\varepsilon$ is the error term;

$\alpha$ picks out any country fixed effects - it is important to account for the fact that each country has different characteristic features and this term holds constant those characteristics that vary across countries but are fixed over time.

\section{Variables used in the analyses}

Health outcome variables: A range of indicators, often split by gender, have been used to capture the health outcome variable. These include life expectancy at birth, life expectancy at age 65, infant mortality, premature mortality, mortality rate, premature mortality from specific diseases and age-adjusted death rate. 
Health input variables: The predominant healthcare input variable used in the literature is healthcare expenditure or healthcare expenditure per capita. In addition, health supply variables such as the number of doctors, hospital beds, type of health system, immunisation coverage, and physicians per capita have also been used in some studies. It is important to note that a healthcare expenditure variable does not explicitly capture either activity or quality aspects of the healthcare system and therefore does not directly answer the question at hand.

Socio-economic and lifestyle variables: Socio-economic factors including education, Gross Domestic Product (GDP) per capita, income distribution, age structure, unemployment rate, pollution, marriage, and crime have been used in the analyses. Individual and lifestyle factors such as tobacco, alcohol and sugar consumption, diet, fruit intake, and pre-existing health conditions have also been used.

\section{The purpose of the studies reviewed}

The primary purpose of the studies reviewed has been to assess the role of healthcare as a determinant of population health outcomes after controlling for socio-economic and individual lifestyle factors. Some have tested for differences in value for money and effectiveness of healthcare across OECD countries.

Three points worth noting here are:

1. Time lag: Most studies have ignored the time lag problem - improvements in medical care might only show effects on mortality after a certain period of time.

2. Disease incidence: Only a very small proportion of studies have taken into consideration the issue of disease incidence. It is important to account for this since the decline in amenable mortality could be due to a decline in incidence of disease as opposed to a decline in case fatalities. Treurniet et al. (1999) have shown that regional variations in mortality in the Netherlands were partly explained by disease incidence variations and that incidence-adjusted mortality rates might be more suited to explain variations in healthcare quality across regions.

3. Use of healthcare expenditure variable: Most studies reviewed have used healthcare expenditure as the main healthcare input variable in their analyses. In addition to not directly answering the question at hand, this poses a methodological problem - higher expenditure could often be found in areas with high mortality rates because resources are diverted to such areas. This makes it harder to separate cause from effect and therefore needs to be taken into account while estimating the relationship. Only a few studies have utilised the appropriate methodology to tackle this problem.

\section{Evidence from pooled time series analyses across OECD countries}

The studies discussed in this section will demonstrate that the evidence that variations in health outcomes can be explained by variations in health inputs is mixed.

Some studies found a weak or no association between the healthcare input variables and health outcomes (Mackenbach 1991; Poikolainen and Eskola 1988; Kunst et al. 1988). For instance, Mackenbach (1991) found no association between per capita healthcare expenditure and GDP- 
adjusted average SMR (Standardised Mortality Rates) from amenable conditions for 11 European Community countries between 1980 and 1984 .

Others found a statistically significant and negative relationship between healthcare variables and mortality indicators (Macinko et al. 2003; Joumard et al. 2008) while some found that socioeconomic factors and/or individual lifestyle factors (Arah et al. 2005; Or 2000) have a higher magnitude of impact:

- One study on the impact of primary care systems on health outcomes (Macinko et al. 2003) in OECD countries found that the strength of a country's primary care system is negatively associated with all-cause premature mortality and cause-specific premature mortality (asthma and bronchitis, emphysema and pneumonia, cardiovascular disease, and heart disease) even after controlling for macro-level (GDP per capita, total physicians per 1,000 population, per cent of elderly) and micro-level (average number of ambulatory care visits, per capita income, alcohol and tobacco consumption) determinants of health. Primary care system characteristics were assessed using a common set of indicators - regulation, financing, primary care provider, access, longitudinally, first contact, comprehensiveness, coordination, family-centred, community-oriented.

- In another study, though healthcare coverage, immunisation and collective health expenditure were found to have negative effects on mortality and premature death, it was alcohol, tobacco and fat consumption that were significantly associated with higher all-cause mortality and premature death (Arah et al. 2005)

- The importance of socio-economic factors is demonstrated in a study (Or 2000) that finds the rise in the employment share of white collar workers and the rise in per capita income to have played the greatest role in the reduction of premature mortality between 1970 and 1992 across 21 OECD countries

\section{Evidence from country-specific analyses}

The studies reviewed in this section are restricted to analyses within a country and often attempt to explain subnational variations. Most found that socio-economic factors more than healthcare variables explain the decline in amenable mortality.

\section{Evidence from OECD countries}

A study of the USA (Thornton 2002) showed that additional medical care utilisation was relatively ineffective in lowering mortality and increasing life expectancy and the most important factors that influenced death rates related to socio-economic status and lifestyle. Similarly, studies of France (Jougla et al. 1987) and the Netherlands (Mackenbach et al. 1988) showed weak and inconsistent relationships between health status and health supply variables.

\section{Evidence from the UK}

Studies of the UK on the impact of the NHS on reducing amenable mortality are scarce. Earlier studies of England and Wales (Bauer and Charlton 1986; Charlton et al. 1983) did not directly test for the relationship between healthcare input and healthcare outcomes (Carr-Hill et al.1987). 
One recent study (Martin et al. 2008) has tackled the inherent methodological problem while estimating a health production function - the need to account for the fact that higher expenditure is often found in areas with higher mortality rates. The main aim of this paper was to determine if extra spending gives rise to better health outcomes, after controlling for need. In that respect, the study does not test the relationship between amenable mortality and healthcare activity per se. Their results, however, show that healthcare expenditure has a positive effect on outcomes in five care programmes - cancer, circulation problems, respiratory problems, gastro-intestinal problems and diabetes. This study is a starting point for future research on amenable mortality in the UK - if the methodology is applied to test the relationship between mortality from amenable conditions and healthcare activity and quality, the findings would greatly reduce the current gap in the literature.

\section{What the empirical analyses tell us}

No study reviewed so far has explicitly used a healthcare activity or quality variable in their analyses. Therefore, it is rather difficult to draw definitive conclusions about the relationship between amenable mortality and the healthcare system. In addition, there is a lack of discussion in the literature about what variables could be used to capture healthcare activity and quality. Healthcare expenditure and supply variables can act as a proxy for healthcare activity and quality. However, studies that tend to use this variable are attempting to answer a different question value for money. Additionally, since most studies have not corrected the inherent methodological problem while estimating a health production function using healthcare expenditure, it is harder to draw clear conclusions.

\section{Conclusions}

There is no consensus in the literature on exactly what constitutes amenable mortality, thereby making the concept in itself imprecise. However, there has been a clear fall in amenable mortality in the past few decades in most OECD countries including the UK. Since the fall in amenable mortality has been at a faster rate than that of unavoidable mortality, some studies have directly attributed this to the healthcare system. Others have tested the relationship between healthcare outcomes and healthcare inputs to determine the magnitude and direction of the relationship.

Since no study has explicitly used a healthcare activity or quality variable in their analyses, the evidence that amenable mortality is an indicator of healthcare quality is far from overwhelming or clear. Though healthcare expenditure can implicitly capture healthcare activity and healthcare quality, the use of this variable is more appropriate to determine value for money across regions. In order to test the relationship between healthcare input and amenable mortality, variables that are directly related to activity and quality of healthcare are essential. It would be ideal if disease incidence is also taken into account.

The fact that most studies have not corrected the inherent methodological problem in estimating a health production function using health expenditure might explain the commonly reported weak and inconsistent results for the healthcare input variables. Though there is strong evidence in the literature for socio-economic and individual lifestyle factors, the magnitude of the impact might be reduced with appropriate methodology and model specification. 
At this stage, it is premature to use amenable mortality in ONS's healthcare output calculations. We welcome comments from those interested in this field, and suggestions to improve understanding in this area. 


\section{References}

Arah O A, Westert G P, Delnij D M and Klazinga N S (2005) 'Health system outcomes and determinants amenable to public health in industrialised countries: a pooled, cross-sectional time series analysis', BMC Public Health, 5:81

Atkinson T (2005) Atkinson Review: Final Report. Measurement of Government Output and Productivity for the National Accounts, Palgrave Macmillan: Basingstoke, 49-50, 29 Jan 2010, available at:

www.statistics.gov.uk/about/data/methodology/specific/PublicSector/Atkinson/final_report.asp

Bauer R L and Charlton J R H (1986) 'Area variation in mortality from diseases amenable to medical intervention: the contribution of differences in morbidity', International Journal of Epidemiology, 15

Bunker J P (2001) 'The role of medical care in contributing to health improvements within societies', International Journal of Epidemiology, 30, 1260-1263

Carr-Hill R A, Hardman G F and Russel I T (1987) 'Variations in avoidable mortality and variations in health care resource', The Lancet, April 4

Charlton J R H, Hartley R M, Silver R and Holland W (1983) 'Geographical variation in mortality from conditions amenable to medical intervention in England and Wales', The Lancet, March 26

Charlton J R H and Velez R (1986) 'Some international comparisons of mortality amenable to medical intervention', British Medical Journal, 292

Cochrane A L, St.Leger A S and Moore F (1978) 'Health service "input" and mortality "output" in developed countries', Journal of Epidemiology and Community Health, 32, 200-205.

Cremieux P, Ouellette P and Pilon C (1999) 'Health care spending as determinants of health outcomes', Health Economics, 8, 627-639

Cutler D, Deaton A and Lleras-Muney (2006) 'The determinants of mortality', Journal of Economic Perspectives, 20:3, 97-120

Cutler D and Miller G (2005) 'The role of public health improvements in health advances: the twentieth century United States', Demography, 42, 1-22

Department of Health (2007) 'Further developments in measuring quality adjusted healthcare output', Department of Health, available at:

www.dh.gov.uk/en/Publicationsandstatistics/Publications/PublicationsStatistics/DH_079021

Jougla E, Ducimetière P, Bouvier-Colle M H and Hatton F (1987) 'Relation between the level of the development of the care system and the level of "preventable" mortality according to department in France', Revue d'épidémiologie et de santé publique 35(5) 
Joumard I, Andre C, Nicq C and Chatal O (2008) 'Health status determinants: lifestyle, environment, health care resources and efficiency', OECD Economics Working Paper 35

Kunst A, Looman C and Mackenbach J P (1998) 'Medical care and regional mortality differences within the countries of the European Community', European Journal of Population, 4

Macinko J, Starfield B and Shi L (2003) 'Primary care systems and health outcomes in OECD countries', Health Services Research, 38:3

Mackenbach J P (1991) 'Health care expenditure and mortality from amenable conditions in the European Community', Health Policy, 19, 245-255

Mackenbach J P, Bouvier-Colle M H and Jougla E (1990) "Avoidable mortality" and health services: a review of aggregate data studies', Journal of Epidemiology and Community Health, 44, 106-111

Mackenbach J P, Kunst A E, Looman C W N, Habbema J D F and van der Maas P J (1986) 'Regional differences in mortality from conditions amenable to medical interventions in The Netherlands: a comparison of four time periods', Journal of Epidemiology and Community Health, 42, 325-332

Martin S, Rice N and Smith P C (2008) 'The link between health care spending and health outcomes for the new English Primary Care Trusts', Centre for Health Economics Research Paper 42

Nixon J and Ulmann P (2006) 'The relationship between health care expenditure and health outcomes' European Journal of Health Economics, 7, 7-18

Nolte E and McKee M (2008) 'Measuring the health of nations: updating an earlier analysis', Heath Affairs, 27, 1

Nolte E and McKee M (2004) Does Health Care Save Lives? Avoidable Mortality Revisited, The Nuffield Trust

ONS (2010) Public Service Productivity: Health Care, Office for National Statistics, available at: http://www.statistics.gov.uk/CCl/article.asp?ID=2382

ONS (2008) Public Service Productivity: Health Care, Office for National Statistics, available at: www.statistics.gov.uk/cci/article.asp?ID=1922

ONS (2006) Public Service Productivity: Health, Office for National Statistics, available at: www.statistics.gov.uk/cci/article.asp?ID=1429

ONS (2004) Public Service Productivity: Health, Office for National Statistics, available at: www.statistics.gov.uk/cci/article.asp?ID=987

Or Z (2000) 'Determinants of health outcomes in industrialised countries: a pooled, cross-country, time series analysis', OECD Economic Studies, 30 
Page A, Tobias M, Glover J, Wright C, Hetzel D and Fisher E (2006) Australian and New Zealand Atlas of Avoidable Mortality, Adelaide: PHIDU, University of Adelaide

Poikolainen K and Eskola J (1986) 'The effect of health services in mortality: decline in death rates from amenable and non-amenable causes in Finland', The Lancet, January 25

Poikolainen K and Eskola J (1988) 'Health services resources and their relation to mortality from causes amenable to health care intervention: a cross national study', International Journal of Epidemiology, 17

Thorton J (2002) 'Estimating a health production function for the US: some new evidence', Applied Economics, 34, 59-62

Treurniet H, Looman C, van der Maas P J, Mackenbach J P (1999) 'Variations in 'avoidable mortality: a reflection of variations in incidence?', International Journal of Epidemiology, 28, 225232

Wheller L, Baker A, Griffiths C and Rooney C (2007) 'Trends in avoidable mortality in England and Wales, 1993-2005', Health Statistics Quarterly, 34, available at:

www.statistics.gov.uk/CCl/article.asp?|D=1806 